\title{
Broadening of Operating Frequency Band of Magnetic-Type Radio Absorbers by FSS Incorporation
}

\author{
Yuri N. Kazantsev, Alexander V. Lopatin, Natalia E. Kazantseva, Alexander D. Shatrov, Valeri P. Mal'tsev,
} Jarmila Vilcäkovä, and Petr Saha

\begin{abstract}
Problems of the theory of radio absorbers (RAs) involving frequency selective surfaces (FSSs) are considered. A design procedure for these RAs is described that takes into account the multiparameter character of the problem and allows one to determine the optimal characteristics of an FSS that provide the maximal operating bandwidth. RAs for a range of 1-17 GHz are obtained on the basis of polymer composites filled with carbonyl iron and $\mathrm{Co}_{2} \mathrm{Z}$ ferrite, into which an FSS in the form of a biperi- odic array of thin metal rings is embedded. It is shown that the application of such an FSS allows one to increase the operating frequency bandwidth of a magnetic-type RA by a factor of more than 1.5 virtually almost without increasing the thickness of the absorber. Substantially, several types of FSSbased layered magne- todielectric RAs have been produced by compressing molding technique, including a 3-mm-thick RA with an operating bandwidth of

1.5- $\quad 2.7 \mathrm{GHz}$, a 2-mm-thick RA with an operating bandwidth of

1.7- $\quad 4.4 \mathrm{GHz}$, and a 1.6-mm-thick RA with an operating bandwidth of 6.7-16.1 GHz.
\end{abstract}

Index Terms-Electromagnetic interference, electromagnetic shielding, ferromagnetic materials, frequency selective surfaces (FSSs).

ADIO ABSORBING MATERIALS (RAMS) have been the subject of intensive study since the World War II. The first studies were focused on military applications and reached their peak in the 1970-1980s due to the development of the Stealth technology. Recently, there has been a renewal of interest of the scientific community in RAMs, which is associated with an increasing penetration of electronics into all areas of modern life. There are a variety of electron devices that radiate electromagnetic energy into the environment, thus causing many serious problems such as electromagnetic interference (EMI), electromagnetic compatibility (EMC), and a hazardous effect of electromagnetic waves on living organisms. One of the main means for solving these problems is RAMs. The frequency interval of $0.8-10 \mathrm{GHz}$, in which many communication and information-transmission systems operate, is of particular importance. There are a large number of publications [1]-[16] devoted to the design of RAMs in this and other frequency intervals. An RA, which reduces the reflection of incident electromagnetic wave (EMW), represents a layer (layers) of a RAM placed on a metal surface.

Any RA operates in a limited operating frequency band. The usual method for expanding the operating frequency band of an RA consists in using multilayer structures instead of singlelayer ones. However, this increases the thickness and the weight of RAs. Today there exist several leading manufacturers of a wide assortment of RAs, such as the FDK Corporation (Japan) and Laird Technology Company (USA). Thin RAs offered by these manufacturers operate in narrow frequency bands, while broadband RAs have thickness of about 30-60 $\mathrm{mm}$. Thus, the design of thin and simultaneously broadband RAs is a topical problem.

Any single-layer RA is characterized by a certain matching frequency $f_{m}$ and matching thickness $d_{r n}$ for which the reflection coefficient is minimal. The values of $/ \mathrm{m}$ and $\mathrm{dm}$ can be determined either by graphical [16] or analytic [5] methods from the complex permittivity and permeability data. In [5], the values of $f_{m}$ and $d_{m}$ were determined for polymer composites filled with various types of carbonyl iron ( $\mathrm{Cl}$ ). It was shown that the matching frequency can be tuned within a wide range by varying the type and concentration of CI. However, although the frequencies $/ \mathrm{m}$ belong to the relevant microwave band, the absorption bands of the RA are relatively narrow. For instance, the ratio of the extreme frequencies of the operating bands (measured at $-10 \mathrm{~dB}$ level of the reflection coefficient) lies in the interval 1.5-1.8.

One of efficient methods for expanding the range of operating frequencies of RAs is the application of FSSs to the design of RAs [6][14]. The effect of FSSs on the reflection characteristics of an RA based on lossy dielectrics is demonstrated in [6] and [7]. In [8]-[10], the authors considered an RA based on dielectrics and FSSs made of resistive elements. In [11] and

[12] , the authors proposed a microgenetic algorithm (MGA) 


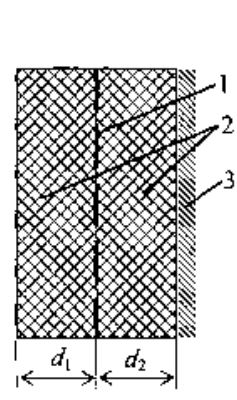

(a)

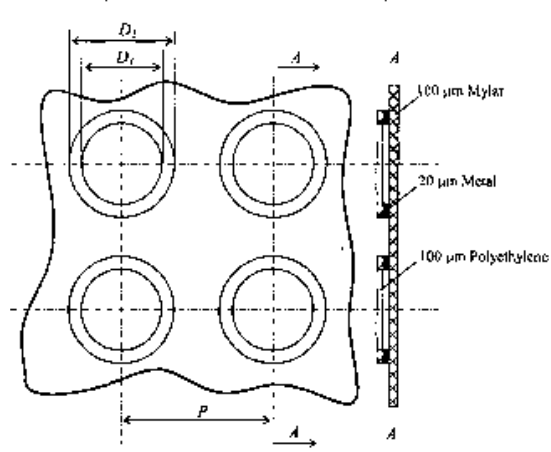

(c)

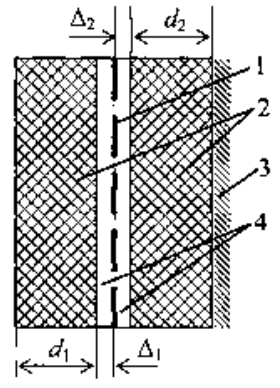

Fig. 1. Radio absorbers with an FSS: (a) FSS embedded into a RAM, (b) FSS is separated from a RAM by dielectric layers, and (c) the FSS structure.

for the design of broadband RAs. In [13], the authors investigated the frequency and angular characteristics of the reflection coefficient of the so-called Circuit Analog Absorbers, which are made of resistive films with frequency selective properties. These authors showed that broadband RAs with small reflection coefficient can be designed for both normal and oblique incidence of TE- and TM-polarized waves. However, since the RAs considered in the above-cited papers were based on FSSs embedded in nonmagnetic materials, they had relatively large thicknesses. In [14], the control of the absorption characteristic of an RA with the use of an FSS is illustrated by an example of polymer composites filled with different types of CL The theoretical methods for studying FSSs (including cascaded FSSs in a dielectric medium) developed earlier in [17]-[23] allow one to calculate the characteristics of RAs with FSSs.

In the present paper, we describe a design procedure for RAs based on polymer magnetic composites that include FSSs. For different regions of the frequency band 1-17 GHz, we use composites filled with different types of $\mathrm{Cl}$ and a ferrite of type $\mathrm{Co} Z \mathrm{Z}$. The measured reflection characteristics are compared with the results of computation by the method of integral equations.

\section{Fundamental Consideration and Analysis}

\section{A. Approximate Theory for the Design of RA With FSS}

Schemes of RAs with FSSs are shown in Fig. 1(a) and (b). In the scheme of Fig. 1(a), an FSS (1) is embedded into the layer of a RAM (2) placed on a metal plane (3). In the scheme of Fig. 1(b), an FSS is separated from the RAM by thin layers (4) of a low-loss dielectric. An FSS in the form of a two-dimensional array of thin metal rings is shown in Fig. 1(c).

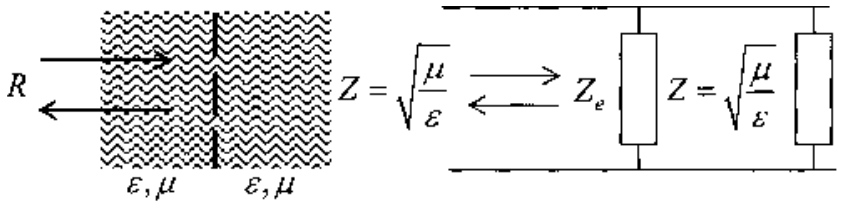

(a)

(b)

Fig. 2. (a) FSS in an infinite magnetodielectric medium, (b) an equivalent circuit.

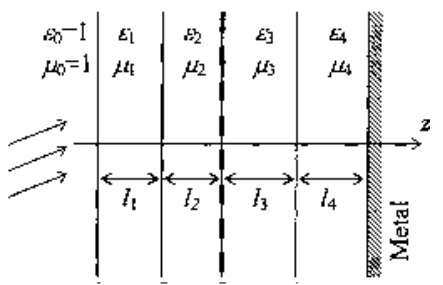

Fig. 3. Multilayered structure of RA with FSS.

When choosing a preliminary structure of an RA, namely, the type of the FSS and the thicknesses $d \pm$ and $\dot{c} 2$ of layers, it is expedient to apply an approximate approach that takes into account the generalized characteristics of an FSS in free space: the resonance frequency $f o$ and the quality factor $Q Q$. In this approach, one first determines the equivalent impedance $Z_{e}$ of the FSS embedded into an infinite magnetodielectric with electric and magnetic losses (Fig. 2(a) and (b)) and then applies the transmission line technique to calculate the reflection coefficient of the structure shown in Fig. 1(a). The formula for the equivalent impedance [14] is expressed as

$$
Z_{e}=\frac{1}{2} \sqrt{\frac{\mu}{\varepsilon}}\left[\frac{Q_{0}}{Q}-1+j Q_{0}\left(\frac{f \operatorname{Re} \sqrt{\varepsilon \mu}}{f_{0}}-\frac{f_{0}}{f \operatorname{Re} \sqrt{\varepsilon \mu}}\right)\right]
$$

where $\varepsilon=\varepsilon^{\prime}-j \varepsilon^{\prime \prime}$ and $\mu=\mu^{\prime}-j \mu^{\prime \prime}$ are complex permittivity and permeability, respectively;

$$
\begin{aligned}
Q & =\frac{Q_{0} Q_{L}}{Q+Q_{L}} \\
Q_{L} & =\frac{\varepsilon^{\prime} \mu^{\prime}}{\varepsilon^{\prime} \mu^{\prime \prime}+\varepsilon^{\prime \prime} \mu^{\prime}} .
\end{aligned}
$$

The reflection coefficient $R$ of the RA with an FSS can easily be obtained by well-known formulas from transmission line theory.

When the FSS is brought close either to the metal plane or to the external surface of the RA, formulas (1)-(3) cease to be valid. For example, when the FSS is placed on the surface, the permittivity and the permeability in these formulas should be replaced by their averaged values $e_{m}=(1+e) / 2$ and $p,{ }_{m}=(1+f j) / 2$.

\section{B. Rigorous Theory}

Consider the problem of reflection of a plane EMW from a multilayer structure shown schematically in Fig. 3. The structure consists of four magnetodielectric layers placed on a metal plane and a planar patch biperiodic metal array enclosed between the second and third layers. 
The method of analysis is based on the numerical solution of an integral equation of the first kind for the surface current in the metal elements of the array. The integral equation is derived by a procedure similar to that used in [17], [22], and [23].

The multilayer structure considered in the present study is different from the structures considered in [22] and [23] both in the position of the FSS in the material and in the presence of a metal plane on the backside of the structure.

Since the integral equation for multilayer structures is too bulky, we will use a recurrence expression for this equation.

Suppose that a plane EMW is incident on the structure. The Cartesian coordinates of the electromagnetic field in this wave are proportional to $\exp \{-j k \cdot \mathbf{r}$ ), where $k$ is the wave vector and the dot denotes the scalar product of vectors. Below, we will denote by a bar below a character two-dimensional transverse vectors that have only $x$ - and y-components. For example, we

denote the projection of $k$ onto the plane $(x, y)$ by $k_{00}$. It is obvious that

$$
\underline{k}_{00}=k \sin \vartheta \cos \varphi \hat{x}+k \sin \vartheta \sin \varphi \hat{y}
$$

where $\hat{e}$ and $i p$ are the polar and azimuth angles that determine the propagation direction of the plane wave and $x^{\wedge} y$ are the unit vectors along the coordinate axes.

The kernel of the integral equation for current in a conducting element of the array is expressed in terms of Floquet functions, which are defined as follows:

$$
\psi_{p q}(x, y)=\exp \left(-j \underline{k}_{p q} \cdot \underline{r}\right)
$$

where

$$
\underline{K}_{p q}=\underline{k}_{00}+\frac{2 \pi}{P_{x}} p \hat{x}+\frac{2 \pi}{P_{y}} q \hat{y} ; \quad p, q=0, \pm 1, \pm 2, \ldots
$$

$P_{x}$ and $P_{y}$ are the dimensions of the unit cell (periods) of the biperiodic array.

Let us introduce two mutually orthogonal unit vectors $n_{l p q}$ and $f \wedge_{2 v q}$ by the formulas

$$
\begin{aligned}
& \underline{\underline{F}}_{1_{\mathcal{T}} q}=\frac{\underline{k}_{P q}}{k_{P q}} \\
& k_{p q}=\left|\underline{k}_{p q}\right| \\
& \underline{\underline{F}}_{2 p q}=\hat{z} \times \underline{\underline{\kappa}}_{1 p q} \text {. }
\end{aligned}
$$

The symbol $\mathbf{x}$ denotes vector multiplication. The transverse coordinates of the electromagnetic field of the Floquet modes propagating in a homogeneous medium with number $n$ are expressed in [17] as

$$
\begin{aligned}
& \underline{E}_{m p q}(x, y, z)=\psi_{m_{q}}(x, y) \exp \left(\mp j \beta_{i q}^{(n)} z\right) \underline{\kappa}_{m p q} \\
& \underline{H}_{m r_{p}}(x, y, z)= \pm \eta_{m p q}^{(n)} \psi_{p q}(x, y) \exp \left(\mp j \rho_{p q}^{(n)} z\right) \hat{z} \times \underline{\kappa}_{m p q}
\end{aligned}
$$

The subscripts $m-1$ and $m=2$ correspond to TM- and TEmodes. The upper and lower signs in (8) correspond to the modes propagating in the positive and negative directions of the axis $z$. The quantities $i 3 p \$$ are propagation constants of the Floquet modes in a magnetodielectric medium with permittivity $e_{n}$ and permeability i.in:

$$
\beta_{p q}^{(n)}=\sqrt{k^{2} \varepsilon_{n} \mu_{n}-k_{p q}^{2}}, \quad \operatorname{In} \beta_{p q}^{(n)}<0
$$

The quantities $r j^{\wedge} p q$ are the characteristic admittances of the traveling Floquet modes:

$$
\begin{aligned}
& \eta_{1 p q}^{(n)}=\frac{k c_{n}}{\beta_{p q}^{(n)}} \\
& \eta_{2 p q}^{(n)}=\frac{\beta_{p q}^{(n)}}{k \mu_{n}} .
\end{aligned}
$$

In the absence of a metal array, the problem of transmission of a Floquet mode with indices $m p q$ through a multilayer magnetodielectric medium is solved easily, because there are no mode conversion at the boundaries of the media and on the metal plane. Therefore, the fields in the nth layer are expressed as

$$
\begin{aligned}
& \underline{E}_{m p q}^{(n)}(x, y, z) \\
& =\left[a^{(n)} \exp \left(-j \beta_{p q}^{(n)} z\right)+b^{(n)} \exp \left(j \beta_{\nu \varphi}^{(n)} z\right)\right] \\
& \times \psi_{p q}(x, y)_{m p q} \\
& \underline{H}_{m p q}^{(n)}(x, y, z) \\
& =\left[\eta_{m p q}^{(n)} a^{(n)} \exp \left(-j \beta_{p q}^{(n)} z\right)\right. \\
& \left.-n_{m p q}^{(n)} b^{(n)} \exp \left(j \beta_{p q}^{(\alpha)} z\right)\right] \psi_{p \psi}(x, y) \hat{z} \times \underline{k}_{m p q}
\end{aligned}
$$

The unknown amplitudes $a$ and of counterpropagating Floquet modes can be determined from a system of algebraic equations that are obtained from the continuity conditions for the vectors $E^{\wedge}$ and $H^{\wedge}{ }_{q}$ on the boundaries between media.

The kernel of the integral equation for current contains terms expressed through the solutions of two auxiliary electrodynamic problems on the propagation of Floquet modes in a multilayer medium without a metal array.

1. Consider a Floquet mode satisfying the condition that the vector $E^{\wedge}{ }_{q}$ vanishes on the metal plane situated on the back side of the multilayer magnetodielectric structure.

Denote by $£ m p q$ the ratio of the field amplitudes and $E_{\mathcal{Y}}^{\mathcal{J}_{m}}$ on the boundary with number $n$ between layers $n-1$ and $n$ It is obvious that

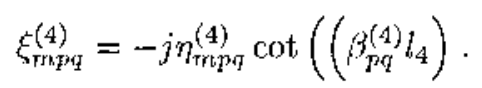

The quantities $£ m p q$ with $n=3,2,1$ are determined by successive application of the descending recurrence formula

$$
\begin{aligned}
\xi_{m p q}^{(n)} & =\eta_{m p q}^{(n)} \\
& \times \frac{\left(\eta_{m p q}^{(n)}+\xi_{m p q}^{(n+1)}\right)-\left(\eta_{m p q}^{(n)}-\xi_{m p q}^{(n+1)}\right) \exp \left(-2 j \beta_{p q}^{(n)} l_{n}\right)}{\left(\eta_{m p q}^{(n)}+\xi_{m p q}^{(n+1)}\right)+\left(\eta_{m p q}^{(n)}-\xi_{m p q}^{(n+1)}\right) \operatorname{cxp}\left(-2 j \beta_{p q}^{(n)} l_{n}\right)}
\end{aligned}
$$


We also introduce

$$
B_{m p q}^{(n)}=\frac{2 r_{m p q}^{(n)} \operatorname{cxp}\left(-j \beta_{p q}^{(n)} l_{n)}\right)}{\left(\eta_{m p q}^{(n)}+\xi_{m p q}^{(n+1)}\right)+\left(n_{m p q}^{(n)}-\xi_{m p q}^{(n+1)}\right) \exp \left(-2 j \beta_{p q}^{(n)} l_{n}\right)}
$$

This function possesses the following property: if we denote by Ampq the amplitude of the electric vector $E^{\wedge}{ }_{q}$ of a standing mode on the boundary with number $n$, then

$$
A_{m p q}^{(r+1)}=B_{r p q}^{(r)} A_{r r q q}^{(n)}
$$

2. Consider another field pattern of the Floquet mode that has a form of a wave propagating in the negative direction of $z$ in the left half-space ( $a=0$ in expression (11)). Denote by -Smpq the ratio of amplitudes of the vectors $K m p q K m l q$ on boundary $n$. On boundary 1 we have

$$
\delta_{\operatorname{mpq}}=\eta_{\mathrm{mpq}}^{(0)}
$$

Here Smpq with $n=2,3,4$ are determined by the following ascending recurrence formula:

$$
\begin{aligned}
& \delta_{r w p q}^{(+1)}=\eta_{r p q}^{(n)}
\end{aligned}
$$

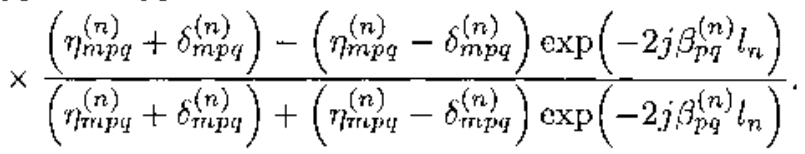

Let us pass on to the problem of diffraction of a Floquet mode by the structure shown in Fig. 3. We will assume that a mode incident on the structure has unit amplitude of the electric field and is characterized by indices $\left(m \backslash p \backslash q^{f}\right)$. Denote by $H_{-} \sim(x, y)$ and $H^{\wedge} \sim(x, y)$ the tangential components of the magnetic field on the left and right of the metal array situated on boundary 3 . In the unit cell of the array, we introduce a vector function $g(x: y)$ by the formula

$$
\underline{H}^{-}(x, y)-\underline{H}^{\dagger}(x, y)=2 \hat{z} \times \underline{g}(x, y) .
$$

The function $g(x, y)$ is proportional to the surface current and is different from zero only in the domain $S^{\prime}$ occupied by metal. Introduce

$$
\tilde{G}_{T h p q}=\frac{1}{P_{u} P_{y}}-\iint_{S} \psi_{p q}^{*}(x, y) \underline{\underline{g}}(x, y) \underline{\kappa}_{r q q q} d x d y
$$

where the asterisk denotes complex conjugation. The required integral equation is expressed as

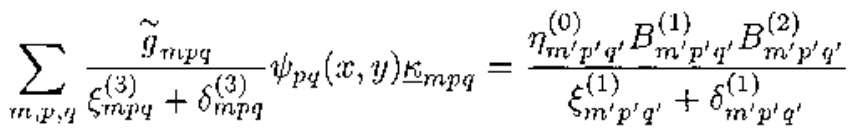

$$
\begin{aligned}
& \times \psi_{p^{\prime} q^{\prime}}(x, y) \kappa_{\mathrm{r}^{\prime} p^{\prime} q^{\prime}}, \quad x, y \in S^{\prime} .
\end{aligned}
$$
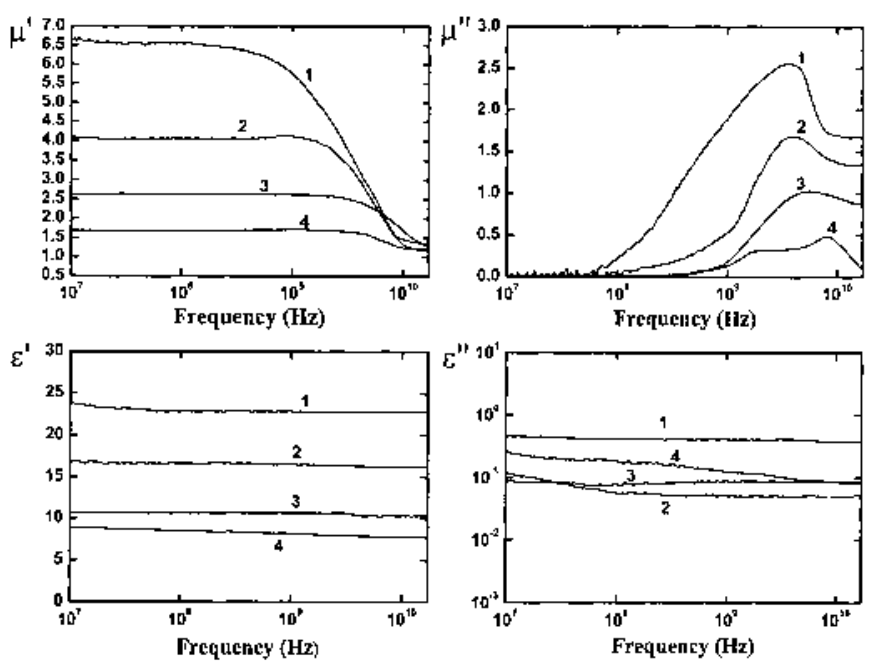

Fig. 4. Electronagnetic characteristics of RAMs; (1) Matbrial I, (2) Material $\Pi$, (3) Material Tח, and (4) Material $T V$.

dices $\mathrm{m}, \mathrm{p}, q$. The amplitudes of these modes are represented in terms of the solution of integral equation (20) by the formula

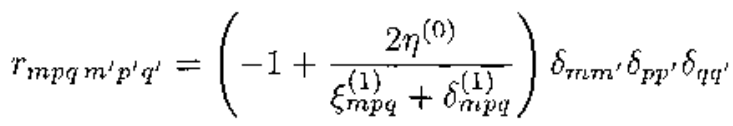

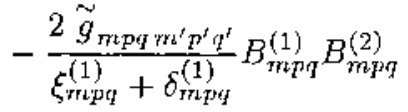

where $6\{j$ is the Kronecker delta.

The components of the matrix of reflection coefficients of a plane incident wave are expressed in terms of $r_{m p q r n} i_{p} i_{q} r$ as follows:

$$
\begin{aligned}
& r_{11}=r_{100100}, \quad r_{12}=\cos \theta r_{200100} \\
& r_{21}=\frac{1}{\cos v} r_{100200}, \quad r_{22}=r_{200200}
\end{aligned}
$$

Here $\mathrm{m}$ and $7^{*} 22$ are the copolarization reflection coefficients, $7^{*} 12$ is the cross-polarization reflection coefficient for a TM-wave, and $7^{*} 21$ is the cross-polarization reflection coefficient for a TE-wave.

Integral equation (20) is solved numerically by the Galerkin method.

\section{An Example of Calculating an RA With an FSS}

The aim of the analysis is to determine the effect of the FSS characteristics (/o, Qo ) and the position of the FSS in the material $\left(i_{1} /{ }^{\wedge} 2\right)^{\text {on }}$ the operating bandwidth of the RA. To this end, we calculated the frequency dependence of the reflection coefficient from the RA for the scheme shown in Fig. 1(a) and for an FSS in the form of a biperiodic array of thin metal rings embedded in a radioabsorbing material representing a polymer composite filled with carbonyl ironMaterial II described in Section III.A. The electromagnetic characteristics of Material II are shown in Fig. 4.

The dimensions $D \pm, D$, and $P$ for several variants of FSS and the values of $d \backslash$ and $<\dot{\dot{c} 2}$ used in the calculations are given in Table I. This table also presents the resonance frequencies

When a plane wave is incident on the structure, the reflected field is expressed as a superposition of Floquet modes with in- 
TABLE I

COMPUTED PARAMETERS OF RA BASED ON MATERIAL II FOR $d=$

\begin{tabular}{|c|c|c|c|c|c|c|c|c|c|c|}
\hline \multirow{2}{*}{$\begin{array}{l}\text { Variant } \\
\text { number }\end{array}$} & \multicolumn{2}{|c|}{$\begin{array}{c}\text { ESS position, } \\
\text { mm }\end{array}$} & \multirow{2}{*}{$\begin{array}{l}P \\
\mathrm{~mm}\end{array}$} & \multirow{2}{*}{$\begin{array}{l}D_{1} \\
\mathrm{~mm}\end{array}$} & \multirow{2}{*}{$\begin{array}{l}D_{2} \\
m \pi\end{array}$} & \multirow{2}{*}{$\begin{array}{l}f_{0} \\
\mathrm{GHz}\end{array}$} & \multirow[t]{2}{*}{$Q_{0}$} & \multirow{2}{*}{$\begin{array}{l}f_{\mathrm{MH}}= \\
\mathrm{GH} x\end{array}$} & \multirow{2}{*}{$\begin{array}{l}f_{\text {itutX }} \\
\mathrm{GHz}\end{array}$} & \multirow{2}{*}{ 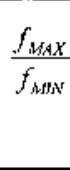 } \\
\hline & $\begin{array}{l}d_{1} \\
\mathrm{~mm}\end{array}$ & $\begin{array}{l}d_{2} \\
\text { man }\end{array}$ & & & & & & & & \\
\hline 1 & \multirow{4}{*}{1.0} & \multirow{4}{*}{1.0} & 9.3 & 5.5 & 6.3 & 19 & 2.8 & - & . & - \\
\hline 2 & & & 6.3 & 3.8 & 4.2 & 28 & 2.8 & - & - & - \\
\hline 3 & & & 5.3 & 3.2 & 3.6 & 33 & 2.8 & 2.84 & 8.14 & 2.87 \\
\hline 4 & & & 4.4 & 2.6 & 3.0 & 40 & 2.8 & $=$ & - & 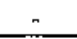 \\
\hline $5^{\mathrm{it}}$ & 1.35 & 0.65 & 2.2 & 16 & 2.1 & 42 & 0.92 & 2.86 & 8.50 & 2.97 \\
\hline 6 & 0.5 & 1.5 & 8.7 & 3.8 & 4.3 & 27 & 6.0 & 2.76 & 7.28 & 2.64 \\
\hline 7 & 0.0 & 2.0 & 16.0 & 5.8 & 6.4 & 17 & 11.9 & 2.71 & 6.71 & 2,48 \\
\hline
\end{tabular}

${ }^{a} D_{1}$ and $D_{2}$ are the inner and outer sides of a square ring, respectively.

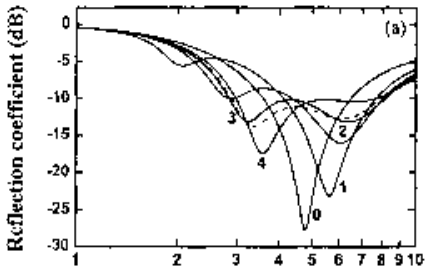

Frequency (GIlly)

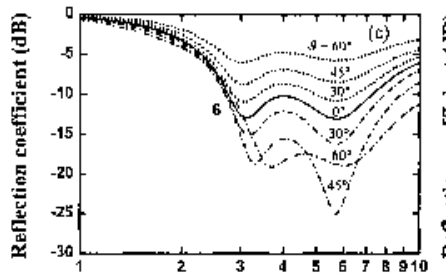

lirequency (GHz)

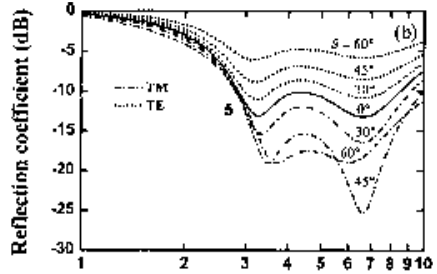

Frequeney (GHz)

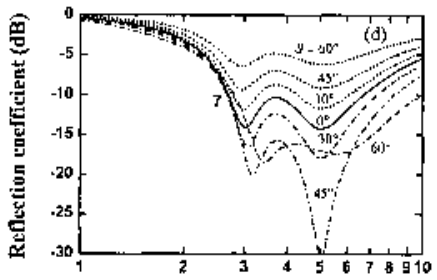

lirequency (GHz)
Fig. 5. Reflection coefficient of RA calculated for normal and oblique incidence of TE and TM polarized waves.

fo and the Q-factors Qo of these FSSs in free space calculated according to [22], as well as the extreme frequencies / $/ \mathrm{min}$ and / $/ \mathrm{max}$ at a level of $-10 \mathrm{~dB}$ of the reflection coefficient.

The results of rigorous calculation of the reflection coefficient from the RA for normal incidence of EMW are shown in Fig. 5(a)-(d) by solid lines. The numbers of curves in the figures correspond to the numbers of variants in Table $I$. The number " 0 " denotes the case when there is no FSS in the composite. This curve attains its minimum at the matching frequency. The dashed curves correspond to variant 3 calculated by the approximate method described in Section II.A.

Fig. 5(a) shows the frequency dependence of the reflection coefficient from the RA for several values of $f o$ and the same values of $Q-2.8$ and $d \bigvee / d_{2}=1$. An increase in the resonance frequency of the FSS leads to a gradual change in the frequency dependence of the reflection coefficient. First, an additional minimum arises in the left part of curve 1, which then moves to the right and simultaneously becomes deeper (see curve 2). In this case, the main minimum slowly moves to the right and becomes shallower. Then both minima become equal in amplitude (see curve 3), in which case the absorption bandwidth at a level of $-10 \mathrm{~dB}$ attains its maximum. The resonance frequency of the FSS embedded in the material is close to the matching frequency of the $\mathrm{RA}, / \mathrm{m}=4.7 \mathrm{GHz}$.
Fig. 5(b)-(d) represent the frequency dependence of the reflection coefficient for three values of $d \backslash /<\dot{2} 2$ when the values of $f o$ and Qo guarantee the maximal operating bandwidth. Calculations show that these values of $f o$ and $Q o$ depend on the ratio $d \pm / \dot{2} 2$ as follows: the greater this ratio, the larger the value of $f o$, and the smaller the Qo For example, when $d \backslash j d z=0, f o=17 \mathrm{GHz}$ and $Q o=11.9$, and when, $i^{1} /^{\wedge} 2=2, f o=42 \mathrm{GHz}$ and $Q o=0.92$. Note that $Q$-factors $>1.2$ can be obtained with an FSS with circular metal rings, while Q-factors smaller than 1.2 can be obtained with an FSS with square rings. The value of $\mathbf{Q o}=0.9$ is the minimum possible value of the $Q$-factor for an FSS with square rings.

According to variants 3, 5, 6 and 7 in Table I, the maximal relative operating bandwidth of the RA weakly depends on the position of the FSS in the material in a rather wide range of values of $\mathbf{d i} / \mathbf{c f e}=\mathbf{0 - 2}$, although any position of the FSS corresponds to specific values of the generalized characteristics of the FSS ( $f o$ and Qo). This situation also occurs with other types of RA made of materials I, III, and IV, whose compositions and characteristics are presented in Section TIT. A.

Fig. 5(b)-(d) also shows the frequency dependence of the reflection coefficient for an oblique incidence of TE- and TM-po- larized waves by dotted line and dash-and-dot lines, respectively. As the angle of incidence \$ increases, the reflection coefficient for TM-polarized waves decreases, whereas that for TE-polarized waves increases. However, the value of the reflection coefficient averaged over both polarizations varies little, up to ' $f f=45^{\circ}$. However, at angles of incidence above $60^{\circ}$, the value of the reflection coefficient for TM-polarized waves starts to increase and approaches unity as $\bullet \& \longrightarrow 90^{\circ}$.

To illustrate the role of FSS in expanding the operating frequency bandwidth of a magnetic-type RA, we consider an absorber structure in which FSS is situated on the surface of the RA (variant 7). Fig. 6 shows the frequency dependence of the equivalent admittance $Y o$ of a layer without an FSS (Fig. 6(a)), equivalent admittance $Y_{e}$ of the FSS on the surface of the layer (Fig. 6(b)), and the sum Yo $+Y_{e}$ (Fig. 6(c)). These functions are also shown in the Smith chart (Fig. 6(d)), where curve 1 corresponds to $Y o$, curve 2 , to $Y_{e}$, and curve 3, to $Y Q+Y_{e}$. At the matching frequency $/ \mathrm{m}$, the imaginary part of $Y o$ vanishes, while its real part is close to unity, which guarantees low reflection coefficient $(-27 \mathrm{~dB})$ at this frequency. The deviation of frequency to either side from $/ \mathrm{m}$ leads to a small variation of 
TABLE II

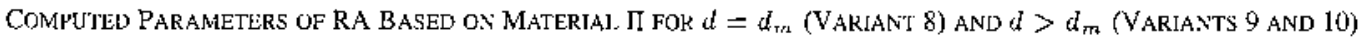

\begin{tabular}{|c|c|c|c|c|c|c|c|c|c|c|c|}
\hline \multirow{2}{*}{$\begin{array}{l}\text { Variant } \\
\text { number }\end{array}$} & \multicolumn{2}{|c|}{$\begin{array}{c}\text { FSS position, } \\
\mathrm{mm} \\
\end{array}$} & \multirow{2}{*}{$\begin{array}{c}P \\
\mathrm{~mm}\end{array}$} & \multirow{2}{*}{$\begin{array}{l}D_{1} \\
\mathrm{~mm}\end{array}$} & \multirow{2}{*}{$\begin{array}{l}D_{2} \\
m m\end{array}$} & \multirow{2}{*}{$\begin{array}{c}f_{0} \\
\mathrm{GH} x\end{array}$} & \multirow[t]{2}{*}{$Q_{0}$} & \multirow{2}{*}{$\begin{array}{l}f_{\text {hiN }}, \\
\mathrm{GHz}\end{array}$} & \multirow{2}{*}{$\begin{array}{l}f_{\operatorname{MAX}} \\
\mathrm{GFIz}\end{array}$} & \multirow{2}{*}{$\frac{f_{M A X}}{f_{M N N}}$} & \multirow{2}{*}{$\begin{array}{l}\text { Reflection } \\
\text { coelhicient in } \\
\text { operating band, } \\
\qquad \mathrm{dB}\end{array}$} \\
\hline & $\begin{array}{l}d_{1}, \\
\text { mint }\end{array}$ & $\begin{array}{l}d_{2} \\
m \mathrm{~m}\end{array}$ & & & & & & & & & \\
\hline 8 & 1.0 & 1.0 & 7.9 & 2.8 & 3.7 & 33 & 7.3 & 3.67 & 5.8 & 1.58 & $\leq-15$ \\
\hline 9 & 1.4 & 1.4 & 9.7 & 3.8 & 4.9 & 25.5 & 5.4 & 2,45 & 4.57 & 1.87 & $\leq-15$ \\
\hline 10 & 1.6 & 1.6 & 12.1 & 4.1 & 5.4 & 22 & 9.1 & 2.37 & 3.52 & 1,49 & $\leq-20$ \\
\hline
\end{tabular}
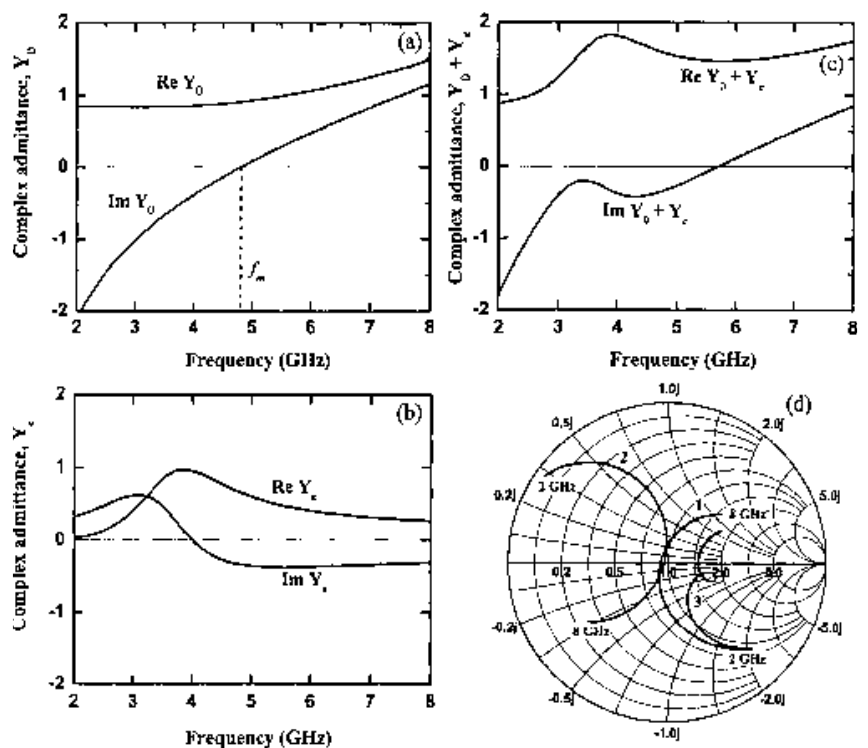

Fig. 6. Equivalent admittance as a function of frequency: (a) admittance of a layer without an FSS $\left(\mathbf{Y}_{0}\right)$, (b) admittance of an FSS on the surface of a layer $\left(\mathbf{Y}_{e}\right)$, (c) the sum of equivalent admittances of the layer and the FSS $\left(Y_{0}+Y_{e}\right)$, and (d) the Smith chart for $Y_{0}$ (1), $Y_{e}(2)$, and $Y_{0}+Y_{e}(3)$.

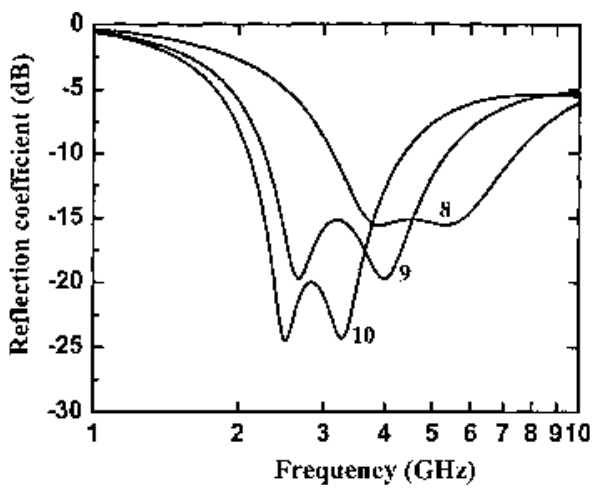

Fig. 7. Reflection coefficient of RA calculated with parameters that guarantee a maximal bandwidth at a reflection coefficient of -15 and $-20 \mathrm{~dB}$.

the real part of admittance; however, the absolute value of the imaginary part $|\operatorname{ImYo}|$ rapidly increases, thus leading to the increase of the reflection coefficient. When an FSS is placed on an RA layer, the admittance of the RA is given by the sum $Y_{0}+Y_{e}$, where the imaginary parts of $Y_{0}$ and $Y_{e}$ have opposite signs and compensate each other. As a result, the imaginary part of the sum $\mathrm{Yo}_{\mathrm{o}}+\mathrm{Y}_{\mathrm{e}}$ approaches zero in a wide frequency range, the operating bandwidth of the RA increases, and the reflection coef ficient as a function of frequency has a shape of a double-hump curve.

Note that one can reduce the reflection coefficient of an RA down to $-20 \mathrm{~dB}$ in a relatively wide frequency band (Table II). Calculations have shown that this can be attained at high Q of the FSS in the following two cases: when $d-d_{m}$ (variant 8 in Table II) and when $d>$ (variants 9 and 10 in Table II). The frequency dependence of the reflection coefficient for these variants is shown in Fig. 7.

\section{Design Procedure of an RA With an FSS}

Now, let us describe the design procedure of an RA with an FSS, which allows considering the multivariable character of the task.

First, we choose an RAM for which the matching frequency lies in the required operating frequency band of the RA. Naturally, the complex permittivity and permeability of this material are already known. The matching frequencies $/ \mathrm{m}$ correspond to matching thicknesses $d_{m}$, which are equal to $d \backslash+d_{2}$. Next, we choose the ratio $d \backslash$ $/ d_{2}$ within the interval 0-2. Applying the transmission-line method, we determine $f o$ and $Q o$ that guarantee the maximal operating bandwidth. The next step consists in choosing the type of a stopband FSS, i.e., choosing the period, as well as the shape and size of individual elements of the two-dimensional array. A mandatory requirement imposed on FSS is the stability of $f o$ and Qo under different polarizations and angles of incidence of an electromagnetic wave. According to [18], an FSS with elements in the form of circular and square rings or Jerusalem crosses exhibits high angular stability. In the case of circular or square rings, the period and the size of elements of the FSS for given values of $f o$ and $Q o$ can be obtained by the methods described in [17]-[22].

The shape of the rings is determined by the required value of Qo For example, a value of Qo $>1.2$ is obtained in FSSs made of circular rings; to obtain $Q_{0}<1.2$, one should use FSSs with square rings.

After evaluating all the basic parameters of the FSS, we should determine them accurately by rigorously calculating the reflection coefficient; in this way we can take into account the effect of gaps between metal elements of the FSS and the RAM. Therewith, to compensate the effect of these gaps, it suffices to reduce the resonance frequency of the FSS by proportionally increasing the dimensions of the FSS structure $\left(D I, D_{2}\right.$, and PI

The procedure described allows one to determine the parameters of the FSS and thus expand the - $10 \mathrm{~dB}-$ bandwidth of a thin single-layer RA of thickness $d m$ matched to free space at 


\begin{tabular}{|c|c|c|c|c|c|c|c|c|c|}
\hline \multirow{2}{*}{$\begin{array}{l}\text { Sample } \\
\text { number }\end{array}$} & \multirow{2}{*}{$\begin{array}{l}\text { Material } \\
\text { number }\end{array}$} & \multirow{2}{*}{$\begin{array}{c}P \\
\mathrm{~mm}\end{array}$} & \multirow{2}{*}{$\begin{array}{c}D_{1} \\
\mathrm{~mm}\end{array}$} & \multirow{2}{*}{$\begin{array}{l}\mathrm{L}_{2} \\
\mathrm{~mm}\end{array}$} & \multicolumn{2}{|c|}{$\begin{array}{c}\text { FSS pasition. } \\
\text { mm }\end{array}$} & \multirow{2}{*}{$\begin{array}{l}f_{\text {MWr }} \\
\mathrm{GHz}\end{array}$} & \multirow{2}{*}{$\begin{array}{l}f_{\text {HAXX }}, \\
\mathrm{GH}:\end{array}$} & \multirow{2}{*}{$\frac{f_{\text {MaK }}}{f_{\text {WNN }}}$} \\
\hline & & & & & $d_{1}$ & $\frac{d_{2}}{\mathrm{~mm}}$ & & & \\
\hline 1 & I & 15.0 & 11.0 & 13.0 & 1.0 & 2,0 & 1.05 & 2.70 & 2.6 \\
\hline 2 & 1 & 15.0 & 8.0 & 10.0 & 1.0 & 2,0 & I .2 & 2.74 & 2.3 \\
\hline 3 & ] & 15.0 & 7.0 & 9.0 & 1.0 & 1.0 & 1.8 & 4.14 & 2.3 \\
\hline 4 & $I$ & 12.0 & 7.0 & 9.0 & 1.0 & 1.0 & 1.7 & 4.4 & 2.6 \\
\hline 5 & \rfloor $I$ & 15.0 & 11.0 & 13.0 & 1.0 & 1.0 & 4.5 & 7.2 & 1.6 \\
\hline 6 & $1 \Gamma$ & 12.0 & 7.0 & 9.0 & 1.0 & 1.0 & 4.8 & 7.6 & 1.6 \\
\hline 7 & II & 12.0 & 5.5 & 7.0 & 1.0 & 1.0 & 3.1 & 7,4 & 2.4 \\
\hline 8 & II & 100 & 5.0 & 6.0 & 1.0 & 1.0 & 3.4 & 5.6 & 1.6 \\
\hline 9 & III & 9.0 & 5.3 & 3.5 & 0.65 & 0.90 & 6.7 & 16.1 & 2.4 \\
\hline 10 & $\mathrm{JV}$ & 13,0 & 4.5 & 6.5 & 1.00 & 2.00 & 5.3 & 9.8 & 1.9 \\
\hline
\end{tabular}

frequency $f_{m}$. If necessary, one can reduce the reflection coefficient of this FSS-based RA down to $-20 \mathrm{~dB}$ in a wide frequency band by optimizing three $\left(\mathrm{Qo}_{\text {? }} / \mathrm{o}>\right.$ and $\left.d\right)$ rather than two (Qo and /o at $d=$ $d_{m}$ ) RA parameters.

\section{in. EXPERIMENTAL SETUP}

\section{A. Materials and Samples}

The basic components for the fabrication of soft magnetic polymer composites are the following fillers: $\mathrm{Cl}$ powders of types $\mathrm{HQ}$ and ES (BASF, Germany), ferrite powders of type $\mathrm{Co}_{2} \mathrm{Z}$ (Ba3Co2Tio.8Fe22.9O41) (Ferrite Domen Co, Russia), glass microspheres of type MSVP A9 (NPO Stekloplastik, Russia), and a polymer binder-a silicon elastomer SYL- GARD 184 (Dow Coming, USA). The glass microspheres were used to improve the homogeneity of the $\mathrm{Cl}$ particle distribution in the composite, as well as to control its complex permittivity. Individual layers of polymer composites were fabricated as follows. First, the basic components were mixed in the required proportion to give a homogeneous mixture. Then, the mixture was placed in a press form and cured for four hours at temperature of $80^{\circ} \mathrm{C}$. After curing, the final product was extracted from the press form.

In this work, we used four types of polymer composites, which had the following compositions and density $p$ :

Material I: 50 vol. \% ES and 50 vol. \% SYLGARD 184, $p=4.43$ $\mathrm{g} / \mathrm{cm}^{3}$;

Material II: 40 vol. \%. HQ, 15 vol. \% MSVP A9, and 45 vol. \% SYLGARD 184, $p=3.64 \mathrm{~g} / \mathrm{cm}^{3}$;

Material III: 30 vol. \%. HQ, 20 vol. \% MSVP A9, and 50 vol. \% SYLGARD 184, $p=2.93 \mathrm{~g} / \mathrm{cm}^{3}$;

Material IV: 50 vol. \% $\mathrm{Co}_{2} \mathrm{Z}$ and 50 vol. \% SYLGARD 184, $p=$ $2.98 \mathrm{~g} / \mathrm{cm}^{3}$.

These polymer composites have low dielectric losses irrespective of the filler type. However, magnetic losses of $\mathrm{Cl}$ filled composites are higher when compared with composites based on $\mathrm{Co2Z}$ (Fig. 4).

The FSS represents a two-dimensional grating of ring-shaped conducting elements (Fig. 1(c)).
The main characteristics of RA samples, as well as the dimensions of the FSS (P, Di, and $\left.D_{2}\right)$ and its position in the RA structure ( $d \pm$ and i2) are shown in Table III.

The RA samples were designed from polymer composite layers and an FSS. The use of several layers of composites of different thickness allowed one to vary both the thickness of a sample and the position of the FSS in the sample.

\section{B. Test Apparatus}

The complex permittivity and permeability of polymer composites were measured by an Agilent E49991A RF Impedance/ Material Analyzer in the frequency range of $0.01-2 \mathrm{GHz}$ and by a resonator method involving $\mathbf{R} 2$ scalar network analyzers, in the frequency range of 2-17 GHz.

The reflection coefficient of the samples was measured by a reflectometer [24] in combination with an HP 8720C Vector Network Analyzer in a laboratory anechoic chamber. To increase the accuracy of these measurements, we applied the technique of subtracting the background level and the time-domain separation of the reflected pulse.

\section{RESUlTS AND Discussion}

The measured reflection coefficients as a function of frequency for ten samples are shown by solid lines in Figs. 8-12. The numbers of the curves in these figures correspond to the numbers of samples in Table III, and the number " 0 " indicates that a sample does not contain an FSS. The sample thicknesses are close to the matching thickness. Fig. 10 clearly illustrates the variation of the frequency dependence of the reflection coefficient as the size of the rings decreases (which corresponds to the increase in the resonance frequency of the FSS). This variation qualitatively reproduces the variations of computed curves in Fig. 5(a). The main difference is that the size of the rings for calculated curves is approximately half that for the measured ones. This result is attributed to the fact that the computations did not take into account the gaps between metal elements of the FSS and the RAM. The results of rigorous calculations, which take into account the above gaps, are shown 


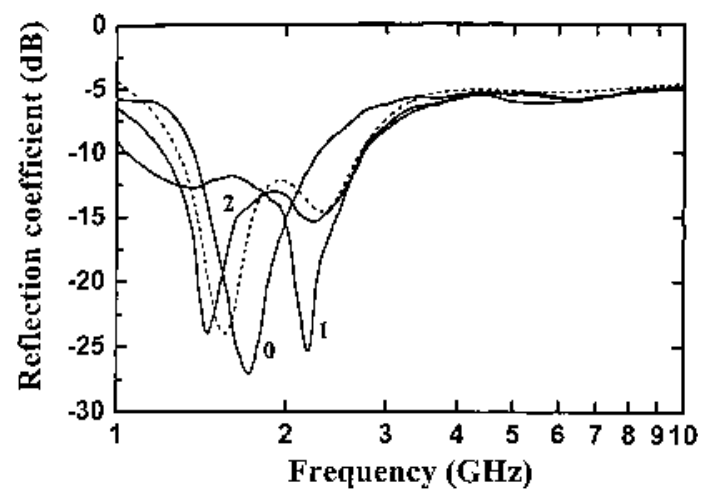

Fig. 8. Measured (solid curves) and computed (dotted curve) reflection coeffi cient versus frequency for samples 1 and 2 .

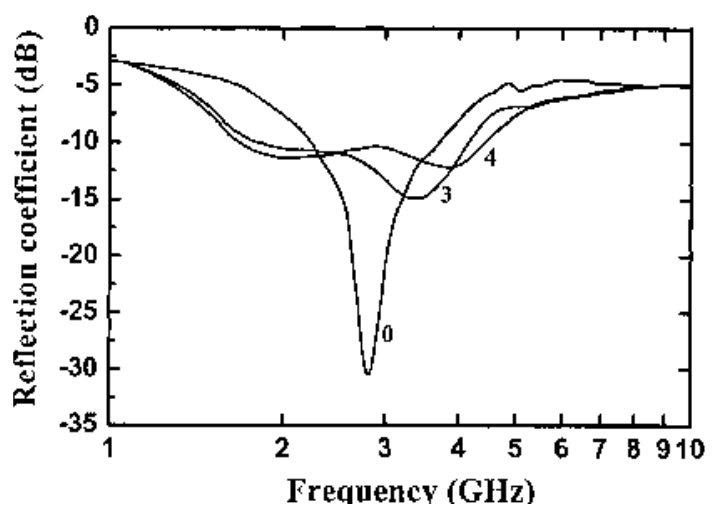

Fig. 9. Reflection coefficient versus frequency for samples 3 and 4.

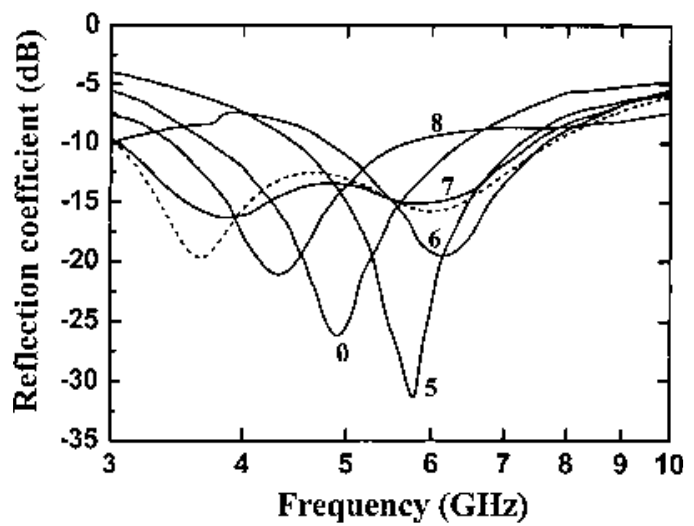

Fig. 10. Measured (solid curves) and computed (dotted curve) reflection coefficient versus frequency for samples 5-8.

by dotted lines in Figs. 8, 10, 11, and 12 for samples 2, 7, 9, and 10, respectively.

The extreme frequencies / $/$ min and / max of the operating frequency bands at a level of $-10 \mathrm{~dB}$ of the reflection coefficient are shown in Table IE. One can see that the type of a material allows one to vary the operating frequency bands of RAs over the region from 1 to $17 \mathrm{GHz}$. For instance, operating frequency bands for samples made of Material I, which is characterized by the maximal values of $e l / /$, and $i j l^{\prime \prime}$, lie in a lower frequency domain than those for samples made of other materials. For example, for sample 1, the operating frequency band is 1.05-2.7 GHz with the ratio of extreme frequencies $/ \max / / \min =2.6$.

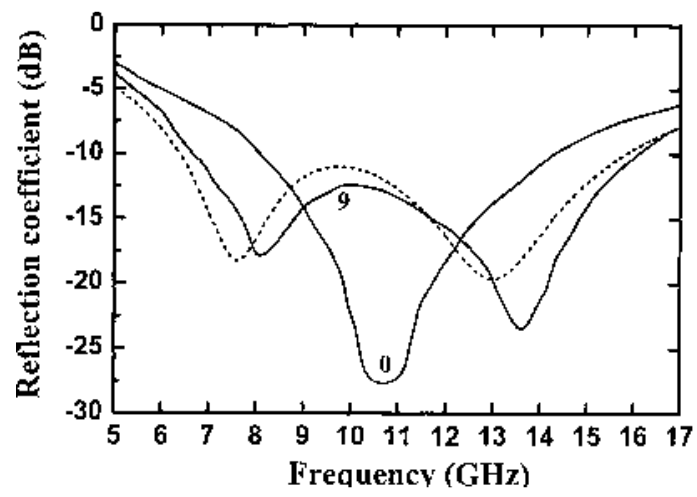

Fig. 11. Measured (solid curves) and computed (dotted curve) reflection coefficient versus frequency for sample 9 .

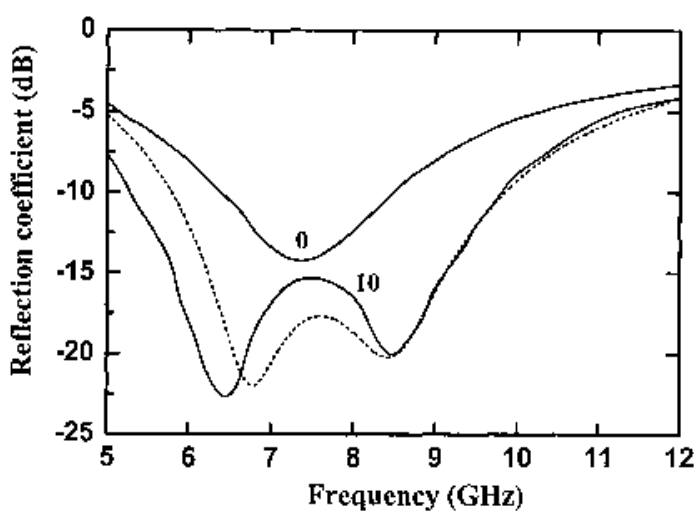

Fig. 12. Measured (solid curves) and computed (dash curve) reflection coefficient versus frequency for sample 10 .

Samples 9 and 10, which are made of Materials IE and IV, respectively, have higher operating frequency bands. For example, the operating frequency band of sample 9 is $6.7-16.1 \mathrm{GHz}$ with the ratio of extreme frequencies 2.4. For samples 1-4, 7, and 9, this ratio lies within the range 2.3-2.6. These results do not contradict the bandwidth estimates by using the data in Table $I$.

\section{Conclusion}

We have demonstrated the efficiency of application of FSSs to controlling the characteristics of thin RAs based on polymer magnetic composites in the range of frequencies from 1 to $17 \mathrm{GHz}$. Two types of Cl that differ in electromagnetic characteristics and a microwave ferrite with Co2Z-type structure have been used as fillers of RAMs.

Approximate analytic expressions for calculating the reflection coefficient from an RA with an FSS and a rigorous theory of multilayer magnetodielectric structures with FSS allow one to carry out a full design of microwave absorbers.

As FSSs, it is recommended to take biperiodic arrays of metal elements in the form of circular or square rings, because the characteristics of these arrays are stable with respect to the angle of incidence of EMW.

In the approximate calculations, we have used generalized characteristics of FSSs (the resonance frequency /o and the Q-factor $Q Q$ ), and the electromagnetic characteristics of the material into which an FSS is embedded. 
The results of rigorous calculation of the reflection coefficient, based on the solution of an integral equation by the Galerkin method, do not contradict the results of calculations obtained by the approximate method.

We have shown that, for any position of the FSS in the RA layer, there exist optimal values of generalized characteristics of the FSS that provide the maximal operating bandwidth at a reflection coefficient of $-10 \mathrm{~dB}$ or lower.

We have proposed a design procedure for thin RA + FSS structures that allows one to take into account a large number of parameters of the problem (the characteristics of the material and the parameters and position of the FSS). First, one determines the thickness of the RA, which is equal to the matching thickness of the given RAM. Then, one chooses the position of the FSS in the RAM layer and determines the generalized characteristics of the FSS, which are optimal for the given position. Finally, one determines the size of the FSS structure by rigorous calculation and makes corrections to take into account the gaps between metal elements of the FSS and the RAM.

Substantially, several types of FSS-based layered magnetodielectric RAs have been produced by compressing molding technique, including a 3-mm-thick RA, a 2-mm-thick RA, and a

1.6- mm-thick RA with $-10 \mathrm{~dB}$ bandwidths of 1.05-2.7 GHz,

1.7- $\quad 4.4 \quad \mathrm{GHz}$, and 6.7-16.1 GHz, respectively.

Thus, we have shown both theoretically and experimentally that the application of an FSS allows one to increase the operating frequency bandwidth of a magnetic-type RA by a factor of more than 1.5 virtually without increasing the thickness of the absorber.

\section{REFERENCES}

[1] J. L. Wallace, "Broadband magnetic microwave absorbers: Fundamental limitation," IEEE Trans. Magn., vol. 29, no. 6, pp. 4209-4214, Nov. 1993.

[2] K. N. Rozanov, "Ultimate thickness to bandwidth ratio of radar absorbers," IEEE Trans. Antennas Propag., vol. 48, no. 8, pp. 1230-1234, Aug. 2000.

[3] M. Amano and Y. Katsuka, "A method of effective use of ferrite for microwave absorber," IEEE Trans. Microw. Theory Tech., vol. 51, no. 1, pp. 238-245, Jan. 2003.

[4] M.-J. Park, J. Choi, and S.-S. Kim, "Wide bandwidth pyramidal absorbers of granular ferrite and carbonyl iron powders," IEEE Trans. Magn., vol. 36, no. 5, pp. 3272-3274, Sep. 2000.

[5] A. V. Lopatin, N. E. Kazantseva, Yu. N. Kazantsev, O. A. D'yakonova, J. Vilcakova, and P. Saha, "The efficiency of application of magnetic polymer composites as radio-absorbing materials," J. Comm. Tech. Electron., vol. 53, no. 5, pp. 487-496, May 2008.

[6] F. Terracher and G. Bergnic, "Thin electromagnetic absorber using frequency selective surface," in Proc. IEEE Antennas Propag. Society Int. Symp., 2000, vol. 2, pp. 846-849.

[7] Y. Sha, K. A. Jose, C. P. Neo, and V. K. Varadan, "Experimental investigation of microwave absorber with FSS embedded in carbon fiber composite," Microw. Opt. Technol. Lett., vol. 32, no. 4, pp. 245-249, Jan. 2002.

[8] L. Hai-Tao, C. Hai-Feng, C. Zeng-Yong, and Z. De-Yong, "Absorbing properties of frequency selective surfaces absorbers with cross-shaped patches," Mater Design, vol. 28, no. 6, pp. 2166-2171, Jun. 2007.

[9] H. Rahman, J. Dowling, and P. K. Saha, "Application of frequency sensitive surfaces in electromagnetic shielding," J. Mat. Proc. Techn., vol. 54, no. 1, pp. 21-28, Oct. 1995.

[10] J. Yang and Z. Shen, "A thin and broadband absorber using double square loops," IEEE Antennas Wireless Propag. Lett, vol. 6, pp. 388-391, Dec. 2007.
[11] S. Chakravarty, R. Mittra, and N. R. Williams, "On the application of the microgenetic algorithm to the design of broadband microwave absorbers comprising frequency-selective surfaces embedded in multilayered media," IEEE Trans. Microw. Tech., vol. 49, no. 6, pp. 1050-1059, Jun. 2001.

[12] S. Chakravarty, R. Mittra, and N. R. Williams, "Application of a micro- genetic algorithm (MGA) to the design of broadband microwave absorbers using multiple frequency selective surface screens buried in dielectrics," IEEE Trans. Antennas Propag., vol. 50, no. 3, pp. 284-296, Mar. 2002.

13] B. A. Munk, P. Munk, and J. Prior, "On designing Jaumann and circuit analog of absorbers (CA absorbers) for oblique angle of incidence," IEEE Trans. Antennas Propag., vol. 55, no. 1, pp. 186-193, Jan. 2007.

[14] A. V. Lopatin, Yu. N. Kazantsev, N. E. Kazantseva, V. N. Apletalin, V. P. Mal'tsev, A. D. Shatrov, and P. Saha, "Radio-absorbing materials on the base of polymer magnetic composites and frequency selective surfaces," J. Comm. Tech. Electron., vol. 53, no. 9, pp. 1114-1122, Sep. 2008.

[15] K. N. Rozanov, Z. W. Li, L. F. Chen, and M. Y. Koledintseva, "Microwave permeability of $\mathrm{Co}_{2} \mathrm{Z}$ composites," J. Appl. Phys., vol. 97, no. 1, pp. 013905-1$013905-7$, Jan. 2005.

[16] Z. W. Li, L. Chen, Y. Wu, and C. K. Ong, "Microwave attenuation properties of W-type barium ferrite $\mathrm{BaZn}_{2}-\mathrm{xCo}_{\mathrm{x}} \mathrm{Fei}_{6} \mathrm{O}_{27}$ composites,” J. Appl. Phys., vol. 96, no. 1, pp. 534-539, Jun. 2004.

[17] J. C. Vardaxoglou, Frequency Selective Surfaces: Analysis and Design. London, U.K.: Research Studies Press, 1997, p. 284.

[18] B. A. Munk, Frequency Selective Surface: Theory and Design.. New York: Wiley, 2000, p. 410.

19] R. Mittra, C. H. Chan, and T. Cwik, "Techniques for analyzing frequency selective surfaces-A review," Proc. IEEE, vol. 76, no. 12, pp. 1593-1615, Dec. 1988.

20] S.-W. Lee, G. Zarrillo, and C.-L. Law, "Simple formulas for transmission through periodic metal grids or plates," IEEE Trans. Antennas Propag., vol. 30, no. 5, pp. 904-909, Sep. 1982.

[21] R. J. Langley and E. A. Parker, "Equivalent circuit model for arrays of square loops,” Electron. Lett., vol. 18, no. 7, pp. 294-296, Apr. 1982.

[22] V. N. Apletalin, Yu. N. Kazantsev, V. P. Mal'tsev, V. S. Solosin, and A. D. Shatrov, "Frequency-selective ring-element gratings," J. Comm. Tech. Electron., vol. 48, no. 5, pp. 469-479, May 2003.

[23] V. N. Apletalin, Yu. N. Kazantsev, V. P. Mal'tsev, and A. D. Shatrov, "A cascade of two island gratings separated by a multilayer magnetodielectric structure," J. Comm. Tech. Electron., vol. 53, no. 6, pp. 631-635, Jun. 2008.

[24] V. N. Apletalin, Yu. N. Kazantsev, and V. S. Solosin, "Microwave and millimeter wave reflectometers based on hollow metal-dielectric waveguides,' Radiotehnika, no. 8, p. 44, Aug. 2005. 